\title{
GLOBAL BIFURCATION FROM INTERVALS IN NONLINEAR STURM-LIOUVILLE PROBLEM WITH INDEFINITE WEIGHT FUNCTION
}

\author{
LEYLA V. NASIROVA
}

\begin{abstract}
We consider the nonlinear Sturm-Liouville problem with indefinite weight and not necessarily differentiable nonlinear term. This problem arise from a selection-migration model in population genetics. The existence of four families of unbounded continua of solutions of this problem having the usual nodal properties and emanating from the bifurcation intervals corresponding to the negative and positive eigenvalues of the linear problem, obtained from nonlinear problem by setting the nonlinear term equal to zero, is proved.
\end{abstract}

\section{Introduction}

We consider the nonlinear Sturm-Liouville problem

$$
\begin{gathered}
\ell(y) \equiv-\left(p(x) y^{\prime}\right)^{\prime}+q(x) y=\lambda \rho(x) y+H\left(x, y, y^{\prime}, \lambda\right), x \in(0,1), \\
\alpha_{0} y(0)-\beta_{0} y^{\prime}(0)=0, \\
\alpha_{1} y(1)+\beta_{1} y^{\prime}(1)=0,
\end{gathered}
$$

where $\lambda \in \mathbb{R}$ is a spectral parameter, the functions $p \in C^{1}[0,1], q, \rho \in C[0,1], p$ is strictly positive and $q$ is nonnegative on $[0,1]$, and $\rho(x)$ is real-valued on $[0,1]$ that take on positive as well as negative values, $\alpha_{i}, \beta_{i}, i=0,1$, are real constants such that $\left|\alpha_{i}\right|+\left|\beta_{i}\right|>0$ and $\alpha_{i} \beta_{i} \geq 0, i=0,1$. The nonlinear term $H$ is of the form $H=f+g$, where $f$ and $g$ are continuous functions on $[0,1] \times \mathbb{R}^{3}$ that satisfy the following conditions:

$$
u f(x, u, s, \lambda) \leq 0, u g(x, u, s, \lambda) \leq 0,(x, u, s, \lambda) \in[0,1] \times \mathbb{R}^{3} ;
$$

there is a positive constant $M$ such that

$$
\begin{gathered}
|f(x, u, s, \lambda) / u| \leq M,(x, u, s, \lambda) \in[0,1] \times \mathbb{R}^{3}, 0<|u| \leq 1,|s| \leq 1 ; \\
g(x, u, s, \lambda)=o(|u|+|s|) \text { as }|u|+|s| \rightarrow 0,
\end{gathered}
$$

uniformly with respect to $(x, \lambda) \in[0,1] \times \Lambda$, for any bounded interval $\Lambda \subset \mathbb{R}$.

Nonlinear eigenvalue problems with indefinite weight arise from a selectionmigration model in population genetics (see, e.g. $[12-14,17,23]$ ). Problem (1.1)-(1.3) for $p(x) \equiv 1, H(x, u, s, \lambda)=\lambda \rho(x)(F(u)-u)$, where $F(u)=u(1-$

2010 Mathematics Subject Classification. 34B15, 34B24, 34C23, 47J10, 47J15.

Key words and phrases. nonlineariar Sturm-Liouville problem, indefinite weight, global bifurcation, usual nodal properties, bifurcation interval. 
$u)\{h(1-u)+(1-h) u\}$ for some constant $h, 0<h<1$, describes the effects of population dispersal within a bounded habitat and selective advantages among the possible genotypes. In this model, weight function $\rho(x)$ that changes sign is a selection coefficient in the habitat, so that a selective advantage at some points of the habitat becomes a disadvantage at others $[13,14]$.

Global bifurcation in nonlinear Sturm-Liouville problem (1.1)-(1.3) with $\rho>0$ have been considered before in $[7,10,19,21,22]$ without conditions $q(x) \geq 0, x \in$ $[0,1], \alpha_{i} \beta_{i} \geq 0, i=0,1$, and condition (1.4). These papers show the existence of unbounded continua of nontrivial solutions in $\mathbb{R} \times C^{1}[0,1]$ that having the usual nodal properties and emanating from bifurcation points and intervals of the line of trivial solutions corresponding to the eigenvalues of the linear problem which obtained from (1.1)-(1.3) by setting $H \equiv 0$. Note that similar results in nonlinear eigenvalue problems for fourth-order ordinary differential equations, elliptic partial differential equations, and Dirac systems were obtained in $[1,5,7$, $19,20]$.

Problem (1.1)-(1.3) was considered in [6] for $f \equiv 0$, and in [4, 18] for $g \equiv 0$. These papers prove the existence of four families of global continua of nontrivial solutions in $\mathbb{R} \times C^{1}[0,1]$ which possesses usual nodal properties and bifurcating from points (for $f \equiv 0$ ) and intervals (for $g \equiv 0$ ) of the line of trivial solutions. It should be noted that in [4] the global bifurcation of solutions from intervals corresponding only to the principal eigenvalues was studied. In [10], it was possible to study the global bifurcation of solutions of (1.1)-(1.3) with $g \equiv 0$ from intervals corresponding to all eigenvalues, but it was not possible to accurately estimate the lengths of these intervals. Similar results in nonlinear eigenvalue problems for fourth-order ordinary differential equations and elliptic partial differential equations with indefinite weights were obtained in $[2,3,8,9,11,12,14,15,17,23$, 24]. In these papers bifurcation of nontrivial solutions were studied from trivial solutions that correspond to the principal eigenvalues of the corresponding linear problems.

In this paper, we are able to estimate the length of the bifurcation intervals corresponding to all eigenvalues of the linear problem obtained from (1.1)-(1.3) by setting $H \equiv 0$ and completely study the unilateral global bifurcation of solutions of problem (1.1)-(1.3).

\section{Preliminary}

As is known [16, Ch. 10], the eigenvalues of the linear spectral problem

$$
\left\{\begin{array}{l}
-\left(p(x) y^{\prime}(x)\right)^{\prime}+q(x) y(x)=\lambda \rho(x) y(x), x \in(0,1), \\
y \in B . C .
\end{array}\right.
$$

where B.C. is the set of functions that satisfy boundary conditions (1.2)-(1.3), are all real and simple, and consist of two unboundedly decreasing and unboundedly increasing sequences $\left\{\lambda_{k}^{-}\right\}_{k=1}^{\infty}$ and $\left\{\lambda_{k}^{+}\right\}_{k=1}^{\infty}$, respectively, such that $\lambda_{1}^{-}<0$ and $\lambda_{1}^{+}>0$. Moreover, for each $k \in \mathbb{N}$ the eigenfunction $y_{k}^{-}(x)\left(y_{k}^{+}(x)\right)$, corresponding to eigenvalue $\lambda_{k}^{-}\left(\lambda_{k}^{+}\right)$, has exactly $k-1$ simple zeros in $(0,1)$.

Remark 2.1. Since the class of continuous functions $C[0,1]$ is dense in $L^{\infty}[0,1]$, the above statements for problem (2.1) also hold for $q \in L^{\infty}[0,1]$. 
Multiplying both sides of (2.1) by $y(x)$ and integrating the result with respect to $x$ from 0 to 1 , using integration by parts, and taking boundary condition (1.2) and (1.3) into account we obtain

$$
\int_{0}^{1}\left\{p(x) y^{\prime 2}(x)+q(x) y^{2}(x)\right\} d x+N[y]=\lambda \int_{0}^{1} \rho(x) y^{2}(x) d x
$$

where

$$
N[y]=-p(1) y^{\prime}(1) y(1)+p(0) y^{\prime}(0) y(0) \geq 0,
$$

in view of conditions $\alpha_{i} \beta_{i} \geq 0, i=0,1$.

Setting $\lambda=\lambda_{k}^{\sigma}$ and $y=y_{k}^{\sigma}(x), k \in \mathbb{N}, \sigma \in\{-,+\}$, in relation (2.2) we get

$$
\int_{0}^{1}\left\{p(x)\left(\left(y_{k}^{\sigma}(x)\right)^{\prime}\right)^{2}+q(x)\left(y_{k}^{\sigma}(x)\right)^{2}\right\} d x+N[y]=\lambda_{k}^{\sigma} \int_{0}^{1} \rho(x)\left(y_{k}^{\sigma}(x)\right)^{2} d x .
$$

Since $\sigma \lambda_{k}^{\sigma}>0$ for $k \in \mathbb{N}, \sigma \in\{-,+\}$, it follows from last equality that

$$
\sigma \int_{0}^{1} \rho(x)\left(y_{k}^{\sigma}(x)\right)^{2}(x) d x>0 .
$$

Let $E$ be the Banach space $E=C^{1}[0,1] \cap B . C$. with the usual norm $\|u\|_{1}=$ $\|u\|_{\infty}+\left\|u^{\prime}\right\|_{\infty}$, where $\|u\|_{\infty}=\max _{x \in[0,1]}|u(x)|$. For each $k \in \mathbb{N}$, each $\sigma \in\{-,+\}$ and each $\nu \in\{-,+\}$ by $S_{k}^{\sigma, \nu}$ we denote be the set of functions $u \in E$ satisfying the following conditions:

1. $u(x)$ has exactly $k-1$ simple zeros in $(0,1)$;

2. $\sigma \int_{0}^{1} \rho(x) u^{2}(x) d x>0$

3. $\lim _{x \rightarrow 0+} \nu \operatorname{sgn} u(x) \rightarrow 1$.

The sets $S_{k}^{-,-}, S_{k}^{-,+}, S_{k}^{+,-}$and $S_{k}^{+,+}$are open subsets of $E$, and $S_{k^{\prime}}^{\sigma^{\prime}, \nu^{\prime}} \cap S_{k}^{\sigma, \nu}$ $=\emptyset$ for $\left(k^{\prime}, \sigma^{\prime}, \nu^{\prime}\right) \neq(k, \sigma, \nu)$. Moreover, if $y \in \partial S_{k}^{\sigma, \nu}$, then either there exists $\tau \in[0,1]$ such that $y(\tau)=y^{\prime}(\tau)=0$, or $\int_{0}^{1} \rho(x) y^{2}(x) d x=0$.

Due to the presence of the nonlinear term $f$, the set of bifurcation points to problem (1.1)-(1.3) through the set $S_{k}^{\sigma, \nu}, k \in \mathbb{N}, \sigma, \nu \in\{-,+\}$, with respect to the line of trivial solutions should be contained in intervals surrounding trivial solutions $\left(\lambda_{k}^{\sigma}, 0\right)$ of this problem (see, e.g $\left.[1,7,21]\right)$.

To clarify the lengths of bifurcation intervals, we need to consider the following linear Sturm-Liouville problems

$$
\begin{gathered}
\left\{\begin{array}{c}
\ell(y)(x)-\lambda \rho(x) y(x)=\mu y(x), x \in(0,1), \\
y \in B . C .
\end{array}\right. \\
\left\{\begin{array}{c}
\ell(y)(x)+\psi(x) y(x)=\lambda \rho(x) y(x), x \in(0,1), \\
y \in B . C .,
\end{array}\right. \\
\left\{\begin{array}{c}
\ell(y)(x)+\psi(x) y(x)-\lambda \rho(x) y(x)=\mu y(x), x \in(0,1), \\
y \in B . C .
\end{array}\right.
\end{gathered}
$$

where $\psi \in C[0,1]$ and $\psi(x) \geq 0$ for $x \in[0,1]$. 
It is known that for each fixed $\lambda \in \mathbb{R}$ the spectrum of problem (2.4) consists of an infinitely increasing sequence of real simple eigenvalues

$$
\mu_{1}(\lambda)<\mu_{2}(\lambda)<\ldots<\mu_{k}(\lambda)<\ldots ;
$$

the eigenfunction $y_{k}(x, \lambda)$ that correspond to the eigenvalue $\mu_{k}(\lambda), k \in \mathbb{N}$, has exactly $k-1$ simple zeros in the interval $(0,1)$. We also note that the eigenvalues $\lambda_{k}^{-}$and $\lambda_{k}^{+}, k \in \mathbb{N}$, of (2.1) are zeros of the function $\mu_{k}(\lambda)$.

Let $\lambda_{k, \psi}^{-}$and $\lambda_{k, \psi}^{+}$be respectively the $k$-th negative and positive eigenvalues of problem (2.5), and let $\mu_{k, \psi}(\lambda), \lambda \in \mathbb{R}$ be the $k$-th eigenvalue of problem (2.6). It is obvious that the eigenvalues $\lambda_{k, \psi}^{-}$and $\lambda_{k, \psi}^{+}, k \in \mathbb{N}$, of (2.5) are zeros of the function $\mu_{k, \psi}(\lambda)$.

Lemma 2.1 [4, Lemma 1]. For each $k \in \mathbb{N}$ the following relations hold:

$$
\begin{aligned}
& \mu_{k}(\lambda) \in C^{\infty}(\mathbb{R}), \quad \frac{d \mu_{k}(\lambda)}{d \lambda}=-\frac{\int_{0}^{1} \rho(x) y_{k}^{2}(x, \lambda) d x}{\int_{0}^{1} y_{k}^{2}(x, \lambda) d x}, \lambda \in \mathbb{R} ; \\
& \mu_{k, \psi}(\lambda) \in C^{\infty}(\mathbb{R}), \frac{d \mu_{k, \psi}(\lambda)}{d \lambda}=-\frac{\int_{0}^{1} \rho(x) y_{k, \psi}^{2}(x, \lambda) d x}{\int_{0}^{1} y_{k}^{2}(x, \lambda) d x}, \lambda \in \mathbb{R},
\end{aligned}
$$

where $y_{k, \psi}(x, \lambda)$ is an eigenfunction corresponding to the eigenvalue $\mu_{k, \psi}(\lambda)$.

Lemma $2.2\left[4\right.$, Lemma 2]. The functions $\lambda \rightarrow \mu_{1}(\lambda)$ and $\lambda \rightarrow \mu_{1, \psi}(\lambda)$ are concave on $\mathbb{R}$.

Remark 2.2. The question of the validity of the statements of Lemma 2.2 for the function $\mu_{k}(\lambda)$ and $\mu_{k, \psi}(\lambda)$ for $k \geq 2$ remains open.

Lemma 2.3 [4, formula (3.3)] For each $k \in \mathbb{N}$ and each $\lambda \in \mathbb{R}$ the following relation holds:

$$
0 \leq \mu_{k, \psi}(\lambda)-\mu_{k}(\lambda) \leq K
$$

where $K=\sup \{\psi(x): x \in[0,1]\}$.

Remark 2.3. By Lemma 2.3 it follows from (2.7) that

$$
\lambda_{k, \psi}^{-} \leq \lambda_{k}^{-}<0 \text { and } 0<\lambda_{k}^{+} \leq \lambda_{k, \psi}^{+} .
$$

Lemma 2.4 [4, Lemma 3] For each $\sigma \in\{+,-\}$ the following formulas holds:

$$
\left|\lambda_{1, \psi}^{\sigma}-\lambda_{1}^{\sigma}\right| \leq \frac{\sigma K \int_{0}^{1}\left(y_{1}^{\sigma}(x)\right)^{2} d x}{\int_{0}^{1} \rho(x)\left(y_{1}^{\sigma}(x)\right)^{2} d x}
$$

Remark 2.4. By Remark 2.2 we cannot state that estimate (2.9) holds for the eigenvalues $\lambda_{k}^{\sigma}$ and $\lambda_{k, \psi}^{\sigma}$ for $k \geq 2$. 


\section{Estimates of the distances between the corresponding eigenvalues of problems (2.1) and (2.4)}

For brevity, we introduce the notations

$$
\tilde{a}_{k}^{\sigma}=\left|\frac{d \mu_{k}\left(\lambda_{k}^{\sigma}\right)}{d \lambda}\right|=\frac{\sigma \int_{0}^{1}\left(y_{k}^{\sigma}(x)\right)^{2} d x}{\int_{0}^{1} \rho(x)\left(y_{k}^{\sigma}(x)\right)^{2} d x}, a_{k}^{\sigma}=\tilde{a}_{k}^{\sigma}+\frac{1}{\tilde{a}_{k}^{\sigma}}, k \in \mathbb{N}, \sigma \in\{-,+\} .
$$

Lemma 3.1 For each $k \in \mathbb{N}$ and each $\sigma \in\{-,+\}$ the following estimate holds:

$$
\operatorname{dist}\left\{\lambda_{k, \psi}^{\sigma}, \lambda_{k}^{\sigma}\right\} \leq K a_{k}^{\sigma} \text {. }
$$

Proof. It follows from (2.8) that (3.1) holds for $k=1$. We will prove the theorem for $k \geq 2$ and $\sigma=+$ (the case of $\sigma=-$ is considered similarly).

It follows from Lemma 2.1 that $\mu_{k}(\lambda)$ and $\mu_{k, \psi}(\lambda)$ are decreasing functions in $(0,+\infty)$. Moreover, $\mu_{k}\left(\lambda_{k}^{+}\right)=0$ and $\mu_{k, \psi}\left(\lambda_{k, \psi}^{+}\right)=0$. By Remark 2.3 we have $0<\lambda_{k}^{+} \leq \lambda_{k, \psi}^{+}$

Let $A=\left(\lambda_{k}^{+}, 0\right), B=\left(\lambda_{k, \psi}^{+}, 0\right), C=\left(\lambda_{k, \psi}^{+}, \mu_{k}\left(\lambda_{k, \psi}^{+}\right)\right)$, and let $\ell_{k}$ be the tangent to the graph of the function $\mu_{k}(\lambda)$ at the point $A, \gamma_{k}$ be the angle formed by the line $\ell_{k}^{+}$and the positive direction of the axis $O \lambda$, and $\tilde{\ell}_{k, \psi}^{+}$be the line parallel to the line $\ell_{k}^{+}$passing through the point $B$. It follows from the first relation of (2.7) that $\gamma_{k}^{+} \in\left(\frac{\pi}{2}, \pi\right)$ and $\tilde{a}_{k}^{+}=-\tan \gamma_{k}$.

Consider two possible cases.

Case 1. Let $\ell_{k}^{+} \cap[B C] \neq \emptyset$, where $[B C]$ is a closed interval connecting points $B$ and $C$ (this case takes place for $k=1$ since the function $\mu_{1}(\lambda)$ is concave on $\mathbb{R})$. By virtue of $(2.8)$ we have $|B C| \leq K$, where $|B C|$ is the length of the closed interval $[B C]$.

Let $D=\ell_{k}^{+} \cap[B C]$. Then

$$
|B D|=|A B| \tan \left(\pi-\gamma_{k}^{+}\right)=-|A B| \tan \gamma_{k}^{+}=|A B| \tilde{a}_{k}^{+},
$$

which implies that

$$
0 \leq \lambda_{k, \psi}^{+}-\lambda_{k}^{+}=|A B|=\frac{|B D|}{\tilde{a}_{k}^{+}} \leq \frac{|B C|}{\tilde{a}_{k}^{+}} \leq \frac{K}{\tilde{a}_{k}^{+}} \leq K a_{k}^{+} .
$$

Case 2. Let $\ell_{k} \cap[B C]=\emptyset$. In this case let $\hat{\ell}_{k, \psi}^{+}$be a line parallel to the axis $O \lambda$ passing through the point $C$. We denote: $E=\hat{\ell}_{k, \psi}^{+} \cap \ell_{k}^{+}$and $F=\hat{\ell}_{k, \psi}^{+} \cap \tilde{\ell}_{k, \psi}^{+}$.

It is obvious that $\angle C B F=\gamma_{k}-\frac{\pi}{2}$, and consequently,

$$
|C F|=|B C| \tan \angle C B F=|B C| \tan \left(\gamma_{k}^{+}-\frac{\pi}{2}\right)=-|B C| \tan ^{-1} \gamma_{k}^{+} \leq \frac{K}{\tilde{a}_{k}^{+}} \text {. }
$$

Let $G \in[A E]$ such that $[B G] \perp[A E]$, and let $\delta_{k}^{+}=\angle E B C$. Note that

$$
0<\delta_{k}^{+} \leq \angle G B C=\angle G B F-\angle C B F=\frac{\pi}{2}-\gamma_{k}^{+}-\frac{\pi}{2}=\pi-\gamma_{k}^{+} \in\left(0, \frac{\pi}{2}\right) .
$$


Then from the right triangle $\triangle E B C$ we get

$$
|E C|=|B C| \tan \delta_{k}^{+} \leq|B C| \tan \left(\pi-\gamma_{k}^{+}\right) \leq-K \tan \gamma_{k}^{+}=K \tilde{a}_{k}^{+} .
$$

Therefore, it follows from (3.2) and (3.3) that

$$
0 \leq \lambda_{k, \psi}^{+}-\lambda_{k}^{+}=|A B|=|E F|=|E C|+|C F| \leq K \tilde{a}_{k}^{+}+\frac{K}{\tilde{a}_{k}^{+}}=K a_{k}^{+} .
$$

The proof of this lemma is complete.

\section{Existence and structure of bifurcation points of problem (1.1)-(1.3) through the set $S_{k}^{\sigma, \nu}$}

We define the norm in $\mathbb{R} \times E$ by $\|(\lambda, y)\|=\left\{|\lambda|^{2}+\|y\|_{1}^{2}\right\}^{\frac{1}{2}}$.

We say that $(\lambda, 0)$ is a bifurcation point of problem (1.1)-(1.3) with respect to the set $\mathbb{R} \times S_{k}^{\sigma, \nu}$, if in every small neighborhood of this point there is a solution to problem (1.1)-(1.3) which is contained in $\mathbb{R} \times S_{k}^{\sigma, \nu}$ (see [1]).

Remark 4.1. The nonlinear eigenvalue problem (1.1)-(1.3) cannot have a nontrivial solution of the form $(0, y) \in \mathbb{R} \times E$. Indeed, multiplying both sides of (1.1) for $\lambda=0$ by $y(x)$ and integrating the result from 0 to 1 , using the formula for the integration by parts, and taking boundary condition (1.2) and (1.3) into account we get

$$
\begin{gathered}
\int_{0}^{1}\left\{p(x) y^{\prime 2}(x)+q(x) y^{2}(x)\right\} d x+N[y]=\int_{0}^{1} f\left(x, y(x), y^{\prime}(x), \lambda\right) y(x) d x+ \\
\int_{0}^{1} g\left(x, y(x), y^{\prime}(x), \lambda\right) y(x) d x,
\end{gathered}
$$

By (2.3) the left hand side of (4.1) is positive, and by (1.4) the right hand side of (4.1) is non-positive, a contradiction.

Remark 4.2. Let $(\lambda, y) \in \mathbb{R} \times E$ be a solution of (1.1)-(1.3) such that $y \in \partial S_{k}^{\sigma, \nu}$. Then by following the arguments in Lemma 4 of [4] with the use of conditions (1.4)-(1.6) we can show that $y \equiv 0$.

In the case of $f \equiv 0$ for (1.1)-(1.3) we have the following global bifurcation result.

Theorem 4.1. For each $k \in \mathbb{N}$, each $\sigma \in\{-,+\}$ and each $\nu \in\{-,+\}$ there is a continuum $C_{k}^{\sigma, \nu}$ of solutions of problem (1.1)-(1.3) with $f \equiv 0$ that contain $\left(\lambda_{k}^{\sigma}, 0\right)$, lies in $\left(\mathbb{R}^{\sigma} \times S_{k}^{\sigma, \nu}\right) \cup\left\{\left(\lambda_{k}^{\sigma}, 0\right)\right\}$, and is unbounded in $\mathbb{R}^{\sigma} \times E$, where $\mathbb{R}^{\sigma}=\{z \in \mathbb{R}: 0<\sigma z<+\infty\}$.

The proof of this theorem is similar to that of [19, Theorem 2.3] with the use of Remarks 4.1 and 4.2.

We introduce the following notation:

$$
b_{k}^{\sigma}=M a_{k}^{\sigma}, J_{k}^{-}=\left[\lambda_{k}^{-}-b_{k}^{-}, \lambda_{k}^{-}\right], J_{k}^{+}=\left[\lambda_{k}^{+}, \lambda_{k}^{+}+b_{k}^{+}\right], k \in \mathbb{N}, \sigma \in\{-,+\} .
$$

For each $k \in \mathbb{N}$, each $\sigma \in\{-,+\}$ and each $\nu \in\{-,+\}$ let $B_{k}^{\sigma, \nu}$ be the set of bifurcation points of problem (1.1)-(1.3) with respect to the set $\mathbb{R} \times S_{k}^{\sigma, \nu}$. The following theorems shows that these sets are not empty and in what intervals they are contained. 
Theorem 4.2. $B_{k}^{\sigma, \nu} \neq \emptyset$ for each $k \in \mathbb{N}$, each $\sigma \in\{-,+\}$ and each $\nu \in\{-,+\}$.

Proof. For the proof of the theorem we will use the approximate problem

$$
\begin{aligned}
& \ell(y)=\lambda \rho(x) y+f\left(x, y|y|^{\varepsilon}, y^{\prime}, \lambda\right)+g\left(x, y, y^{\prime}, \lambda\right), \varepsilon \in(0,1), x \in(0,1), \\
& y \in \text { B.C. }
\end{aligned}
$$

By (1.5) we have

$$
f\left(x, u|u|^{\varepsilon}, s, \lambda\right)=o(|u|+|s|) \text { as }|u|+|s| \rightarrow 0
$$

in the uniform sense of condition (1.6). Hence Theorem 4.1 imply that for each $k \in \mathbb{N}$, each $\sigma \in\{+,-\}$, each $\nu \in\{+,-\}$, and every $\varepsilon \in(0,1)$ there is an unbounded component $D_{k, \varepsilon}^{\sigma, \nu}$ of solutions of problem (4.2) such that

$$
\left(\lambda_{k}^{\sigma}, 0\right) \in C_{k, \varepsilon}^{\sigma, \nu} \subset\left(\mathbb{R}^{\sigma} \times S_{k}^{\sigma, \nu}\right) \cup\left\{\left(\lambda_{k}^{\sigma}, 0\right)\right\} .
$$

We now choose some fixed arbitrary $k_{0} \in \mathbb{N}, \sigma_{0} \in\{-,+\}$ and $\nu_{0} \in\{-,+\}$, and we will prove the theorem for $k=k_{0}, \sigma=\sigma_{0}$ and $\nu=\nu_{0}$.

By (4.3) for every $\varepsilon \in(0,1)$ and each sufficiently small $\tau \in(0,1)$ there is a solution $\left(\lambda_{\varepsilon, \tau, 0}, y_{\varepsilon, \tau, 0}\right)=\left(\lambda_{k_{0}, \varepsilon, \tau}^{\sigma_{0}, \nu_{0}}, y_{k_{0}, \varepsilon, \tau}^{\sigma_{0}, \nu_{0}}\right) \in \mathbb{R}^{\sigma_{0}} \times E$ of problem (4.3) such that

$$
y_{\varepsilon, \tau, 0} \in S_{k_{0}}^{\sigma_{0}, \nu_{0}} \text { and }\left\|y_{\varepsilon, \tau, 0}\right\|_{1}=\tau .
$$

It is obvious that $\left(\lambda_{\varepsilon, \tau, 0}, y_{\varepsilon, \tau, 0}\right)$ solves the following linearizable Sturm-Liouville problem

$$
\begin{aligned}
& \ell y+\psi_{\varepsilon, \tau, 0}(x) y=\lambda \rho(x) y+g(x, y, y, \lambda), x \in(0,1), \\
& y \in B . C .
\end{aligned}
$$

where

$$
\psi_{\varepsilon, \tau, 0}(x)=\left\{\begin{array}{cc}
-\frac{f\left(x, y_{\varepsilon, \tau, 0}(x)\left|y_{\varepsilon, \tau, 0}(x)\right|^{\varepsilon}, y_{\varepsilon, \tau, 0}^{\prime}(x), \lambda_{\varepsilon, \tau, 0}\right)}{y_{\varepsilon, \tau, 0}(x)} & \text { if } y_{\varepsilon, \tau, 0}(x) \neq 0, \\
0, & \text { if } y_{\varepsilon, \tau, 0}(x)=0,
\end{array} \quad x \in[0,1] .\right.
$$

Since $\tau \in(0,1)$, by virtue of (1.4) and (1.5), it follows from (4.6) that

$$
0 \leq \psi_{\varepsilon, \tau, 0}(x) \leq M\left|y_{\varepsilon, \tau, 0}(x)\right|^{\varepsilon} \leq M, x \in[0,1] .
$$

The linearization of (4.5) at $y=0$ is given by

$$
\begin{aligned}
& \ell y+\psi_{\varepsilon, \tau, 0}(x) y=\lambda \rho(x) y, x \in(0,1), \\
& y \in B . C .
\end{aligned}
$$

In view of (4.7), by Remarks 2.1, 2.3 and relation (3.1) we get

$$
\lambda_{k, \psi_{\varepsilon, \tau, 0}}^{\sigma} \in J_{k}^{\sigma}
$$

where $\lambda_{k, \psi_{\varepsilon, \tau, 0}}^{-}$and $\lambda_{k, \psi_{\varepsilon, \tau, 0}}^{+}$are the $k$-th negative and positive eigenvalues of the linear problem (4.8) respectively. By (4.4) it follows from Theorem 4.1 that we can choose a sufficiently small $\varrho_{\varepsilon, \tau}>0$ such that

$$
\left|\lambda_{\varepsilon, \tau, 0}-\lambda_{k, \psi_{\varepsilon, \tau, 0}}^{\sigma_{0}}\right| \leq \varrho_{\varepsilon, \tau} .
$$

Let $\sup _{\varepsilon, \tau} \varrho_{\varepsilon \tau}=\varrho_{0}$. Then the relations (4.9) and (4.10) implies that

$$
\lambda_{\varepsilon, \tau, 0} \in J_{k_{0}}^{\sigma_{0}}\left(\varrho_{0}\right),
$$


where

$$
J_{k}^{-}(\varrho)=\left[\lambda_{k}^{-}-b_{k}^{-}-\varrho, \lambda_{k}^{-}+\varrho\right] \text { and } J_{k}^{+}(\varrho)=\left[\lambda_{k}^{+}-\varrho, \lambda_{k}^{+}+b_{k}^{+}+\varrho\right] .
$$

In view of (4.4) and (4.11), by (1.5), (1.6) it follows from equation in (4.2) that the set $\left\{y_{\varepsilon, \tau, 0} \in E: 0<\varepsilon<1\right\}$ is bounded in $C^{2}[0,1]$. Consequently, by the Arzelà-Ascoli theorem this set is precompact in $E$. Then from any sequence $\left\{\varepsilon_{n}\right\}_{n=1}^{\infty} \subset(0,1)$ converging to zero we can extract a subsequence $\left\{\varepsilon_{n_{m}}\right\}_{m=1}^{\infty} \subset$ $(0,1)$ such that $\left(\lambda_{\varepsilon_{n_{m}}, \tau, 0}, y_{\varepsilon_{n_{m}}, \tau, 0}\right) \rightarrow\left(\lambda_{\tau, 0}, y_{\tau, 0}\right)$ in $\mathbb{R}^{\sigma_{0}} \times E$ as $m \rightarrow \infty$. Equation (4.2) then shows that this subsequence is convergent also in $\mathbb{R} \times C^{2}[0,1]$. Hence setting $\varepsilon=\varepsilon_{n_{m}}, \lambda=\lambda_{\varepsilon_{n_{m}}, \tau, 0}$ and $y=y_{\varepsilon_{n_{m}}, \tau, 0}$ in (4.2), and passing to the limit as $m \rightarrow \infty$ we obtain that $\left(\lambda_{\tau, 0}, y_{\tau, 0}\right)$ is a solution of (1.1)-(1.3) such that $\left\|y_{\tau, 0}\right\|_{1}=\tau$. Then by Remark 4.2 it follows from (4.4) that $y_{\tau, 0} \in S_{k_{0}}^{\sigma_{0}, \tau_{0}}$.

Thus we have shown that for any sufficiently small $\tau>0$ there exists a solution $\left(\lambda_{\tau, 0}, y_{\tau, 0}\right) \in \mathbb{R} \times E$ of problem (1.1)-(1.3) such that

$$
\lambda_{\tau, 0} \in J_{k_{0}}^{\sigma_{0}}\left(\varrho_{0}\right),\left\|y_{\tau, 0}\right\|_{1}=\tau \text {, and } y_{\tau, 0} \in S_{k_{0}}^{\sigma_{0}, \nu_{0}} .
$$

Then any sequence $\left\{\left(\lambda_{\tau_{n}, 0}, y_{\tau_{n}, 0}\right)\right\}_{n=1}^{\infty} \subset \mathbb{R} \times E$, where $\tau_{n}>0, n \in \mathbb{N}$, and $\tau_{n} \rightarrow 0$ as $n \rightarrow \infty$, of solutions to problem (1.1)-(1.3), which satisfies (4.12), contains a subsequence $\left\{\left(\lambda_{\tau_{n_{l}}, 0}, y_{\tau_{n_{l}}, 0}\right)\right\}_{l=1}^{\infty}$ converging to $\left(\lambda_{0}, 0\right)$ for some $\lambda_{0} \in J_{k_{0}}^{\sigma_{0}}\left(\varrho_{0}\right)$. It means that $\left(\lambda_{0}, 0\right) \in J_{k_{0}}^{\sigma_{0}}\left(\varrho_{0}\right) \times\{0\}$ is a bifurcation point of problem (1.1)-(1.3) with respect to the set $\mathbb{R} \times S_{k_{0}}^{\sigma_{0}, \nu_{0}}$. The proof of this theorem is complete.

Theorem 4.3. $B_{k}^{\sigma, \nu} \subseteq J_{k}^{\sigma} \times\{0\}$ for each $k \in \mathbb{N}$, each $\sigma \in\{-,+\}$ and $\nu \in$ $\{-,+\}$.

Proof. By virtue of Remark 4.1 and Theorem 4.2, to prove the theorem it suffices to show that for each $k \in \mathbb{N}$, each $\sigma \in\{-,+\}$ and each $\nu \in\{-,+\}$ the following relation holds:

$$
B_{k}^{\sigma, \nu} \cap\left(\mathbb{R}^{\sigma} \backslash J_{k}^{\sigma}\right)=\emptyset
$$

We again choose arbitrarily fixed $k_{0} \in \mathbb{N}, \sigma_{0} \in\{-,+\}$ and $\nu_{0} \in\{-,+\}$. If (4.13) does not hold for $k=k_{0}, \sigma=\sigma_{0}$ and $\nu=\nu_{0}$, then there exists a sequence $\left\{\left(\lambda_{n, 0}^{*}, y_{n, 0}^{*}\right)\right\}_{n=1}^{\infty} \subset \mathbb{R} \times E$ of solutions of problem (1.1)-(1.3) such that

$$
\left(\lambda_{n, 0}^{*}, y_{n, 0}^{*}\right) \rightarrow\left(\lambda_{0}^{*}, 0\right) \text { as } n \rightarrow \infty, y_{n, 0}^{*} \in S_{k_{0}}^{\sigma_{0}, \nu_{0}}, \lambda_{0}^{*} \notin J_{k_{0}}^{\sigma_{0}} \subset \mathbb{R}^{\sigma_{0}} .
$$

Then we can choose a sufficiently small $\epsilon_{0}>0$ such that

$$
\lambda_{n, 0}^{*} \notin J_{k_{0}}^{\sigma_{0}}\left(\epsilon_{0}\right) \text { for a sufficiently large } n \in \mathbb{N} \text {. }
$$

It is obvious that $\left(\lambda_{n, 0}^{*}, y_{n, 0}^{*}\right), n \in \mathbb{N}$, solves the nonlinear eigenvalue problem

$$
\begin{aligned}
& \ell y+\psi_{n, 0}^{*}(x) y=\lambda \rho(x) y+g\left(x, y, y^{\prime}, \lambda\right), x \in(0,1), \\
& y \in B . C .
\end{aligned}
$$

where

$$
\psi_{n, 0}^{*}(x)=\left\{\begin{array}{cl}
-\frac{f\left(x, y_{n, 0}^{*}(x),\left(y_{n, 0}^{*}\right)^{\prime}(x), \lambda_{n, 0}^{*}\right)}{y_{n, 0}^{*}(x)} & \text { if } y_{n, 0}^{*}(x) \neq 0, \\
0, & \text { if } y_{n, 0}^{*}(x)=0,
\end{array} \quad x \in[0,1] .\right.
$$

In view of (1.6), we see from Theorem 4.1 that

$$
\left|\lambda_{n, 0}^{*}-\lambda_{k, \psi_{n, 0}^{*}}^{\sigma_{0}}\right|<\epsilon_{0} \text { for a sufficiently large } n \in \mathbb{N},
$$


where $\lambda_{k, \psi_{n, 0}^{*}}^{+}$and $\lambda_{k, \psi_{n, 0}^{*}}^{-}$are the $k$-th negative and positive eigenvalues, respectively, of the linear spectral problem

$$
\begin{aligned}
& \ell y+\psi_{n, 0}^{*}(x) y=\lambda \rho(x) y, x \in(0,1), \\
& y \in B . C .
\end{aligned}
$$

By (1.4) and (1.5) it follows from (4.17) that

$$
0 \leq \psi_{n, 0}^{*}(x) \leq M \text { for } x \in[0,1] \text { and } n \in \mathbb{N} .
$$

Consequently, Remark 2.1 and relation (3.1) implies that

$$
\lambda_{k, \psi_{n, 0}^{*}}^{\sigma_{0}} \in J_{k_{0}}^{\sigma_{0}} .
$$

In view of (4.18), by (4.20) for a sufficiently large $n \in \mathbb{N}$ we get $\lambda_{n, 0}^{*} \in J_{k_{0}}^{\sigma_{0}}\left(\epsilon_{0}\right)$ in contradiction with the relation (4.15). The proof of this theorem is complete.

\section{Unilateral global bifurcation of solutions of problem (1.1)-(1.3)}

Let $\mathcal{D}$ be the closure in $\mathbb{R} \times E$ of the set of nontrivial solutions to the problem (1.1)-(1.3). For each $k \in \mathbb{N}$, each $\sigma \in\{-,+\}$ and each $\nu \in\{-,+\}$ by $\hat{D}_{k}^{\sigma, \nu}$ we denote the union of all the connected components $D_{k, \lambda}^{\sigma, \nu}$ of $\mathcal{D}$ bifurcating from points $(\lambda, 0) \in J_{k}^{\sigma} \times\{0\}$ with respect to the set $S_{k}^{\sigma, \nu}$. By [21, Theorem 3.1] and Theorems $4.2,4.3$ we have $\hat{D}_{k}^{\sigma, \nu} \neq \emptyset$. Note that $D_{k}^{\sigma, \nu}=\hat{D}_{k}^{\sigma, \nu} \cup\left(J_{k}^{\sigma} \times\{0\}\right)$ is a connected subset of $\mathbb{R} \times E$, but $\hat{D}_{k}^{\sigma, \nu}$ may not be connected in $\mathbb{R} \times E$.

The main result of this paper is the following theorem.

Theorem 5.1. For each $k \in \mathbb{N}$, each $\sigma \in\{-,+\}$ and each $\nu \in\{-,+\}$ the set $D_{k}^{\sigma, \nu}$ lies in $\left(\mathbb{R}^{\sigma} \times S_{k}^{\sigma, \nu}\right) \cup\left(J_{k}^{\sigma} \times\{0\}\right)$ and is unbounded $\mathbb{R} \times E$.

Proof. Since $D_{k}^{\sigma, \nu}$ for each $\nu \in\{-,+\}$ is a connected component of $\mathcal{D}$ emanating from $J_{k}^{\sigma} \times\{0\} \subset \mathbb{R}^{\sigma} \times\{0\}$ it follows from Remark 4.1 that $D_{k}^{\sigma,-}, D_{k}^{\sigma,+} \subset \mathbb{R}^{\sigma} \times E$. Moereover, in view of Remark 4.2 and Theorem 4.3 we obtain $\left(D_{k}^{\sigma, \nu} \backslash\left(J_{k}^{\sigma} \times\{0\}\right)\right) \cap$ $\left(\mathbb{R}^{\sigma} \times \partial S_{k}^{\sigma, \nu}\right)=\emptyset$, which implies that $D_{k}^{\sigma, \nu} \subset\left(\mathbb{R}^{\sigma} \times S_{k}^{\sigma, \nu}\right) \cup\left(J_{k}^{\sigma} \times\{0\}\right)$.

Next we choose some fixed arbitrary $k_{0} \in \mathbb{N}, \sigma_{0} \in\{-,+\}$ and $\nu_{0} \in\{-,+\}$, and we will prove that $D_{k}^{\sigma, \nu}$ is unbounded in $\mathbb{R} \times E$ for $k=k_{0}, \sigma=\sigma_{0}$ and $\nu=\nu_{0}$.

If $D_{k_{0}}^{\sigma_{0}, \nu_{0}}$ is bounded in $\mathbb{R} \times E$, then $D_{k_{0}}^{\sigma_{0}, \nu_{0}}$ is precompact in $\mathbb{R} \times E$ in view of (1.1)-(1.3). Hence by following the arguments in Lemma 1.2 of [19] we can find a neighborhood $Q_{0} \subset \mathbb{R}^{\sigma_{0}} \times E$ of this set such that $D_{k_{0}}^{\sigma_{0}, \nu_{0}} \cap \partial Q_{0}=\emptyset$ (see also [7]). Since $D_{k_{0}, \varepsilon}^{\sigma_{0}, \nu_{0}}$ is an unbounded connected component of solutions of (4.2) satisfying (4.3) it follows that for any $\varepsilon_{n} \in(0,1)$ there exists $\left(\lambda_{\varepsilon, 0}, y_{\varepsilon, 0}\right) \in D_{k_{0}, \varepsilon}^{\sigma_{0}, \nu_{0}} \cap \partial Q_{0}$. Then, as in the proof of Theorem 4.1, we can show that there is a sequence $\left\{\varepsilon_{n}\right\}_{n=1}^{\infty} \subset(0,1)$ converging to 0 as $n \rightarrow \infty$ such that $\left(\lambda_{\varepsilon_{n}, 0}, y_{\varepsilon_{n}, 0}\right)$ converges to a solution $\left(\lambda_{0}, y_{0}\right)$ of problem (1.1)-(1.3), and consequently, $y_{0} \in S_{k_{0}}^{\sigma_{0}, \nu_{0}} \cup \partial S_{k_{0}}^{\sigma_{0}, \nu_{0}}$. If $y_{0} \in \partial S_{k_{0}}^{\sigma_{0}, \nu_{0}}$, then it follows from Remark 4.2 that $y_{0} \equiv 0$. By following the arguments in the proof of Theorem 4.2 we get $\lambda_{0} \in J_{k_{0}}^{\sigma_{0}}$ which contradicts the condition $J_{k_{0}}^{\sigma_{0}} \cap \partial Q_{0}=\emptyset$. Hence we have $y_{0} \in S_{k_{0}}^{\sigma_{0}, \nu_{0}}$ in contradiction with the condition $D_{k_{0}}^{\sigma_{0}, \nu_{0}} \cap \partial Q_{0}=\emptyset$. The proof of this theorem is complete. 
If $g \equiv 0$ and $f$ satisfies condition (1.5) for all $(x, u, s, \lambda) \in[0,1] \times \mathbb{R}^{3}$, then for problem (1.1)-(1.3) we get the following global bifurcation result.

Theorem 5.2. Let $g \equiv 0$ and condition (1.5) is satisfied for all $(x, u, s, \lambda) \in$ $[0,1] \times \mathbb{R}^{3}$. Then for each $k \in \mathbb{N}$, each $\sigma \in\{+,-\}$ and each $\nu \in\{+,-\}$ the set $D_{k}^{\sigma, \nu}$ lies in the strip $\left(J_{k}^{\sigma} \times S_{k}^{\sigma, \nu}\right) \cup\left(J_{k}^{\sigma} \times\{0\}\right)$ and is unbounded in $\mathbb{R} \times E$.

The proof of this theorem follows from Theorem 5.1, taking into account the following statement.

Lemma 5.1. Let the conditions of Theorem 5.2 be satisfied and let $\left(\lambda^{*}, y^{*}\right) \in$ $\mathbb{R} \times E$ be a solution to problem (1.1)-(1.3) such that $y^{*} \in S_{k}^{\sigma, \nu}, k \in \mathbb{N}$ and $\sigma, \nu \in\{+,-\}$. Then $\lambda^{*} \in J_{k}^{\sigma}$.

Proof. Let $\left(\lambda^{*}, y^{*}\right) \in \mathbb{R} \times E$ be a solution to problem (1.1)-(1.3) such that $y^{*} \in S_{k_{0}}^{\sigma_{0}, \nu_{0}}$ for some fixed arbitrary $k_{0} \in \mathbb{N}, \sigma_{0} \in\{-,-+\}$ and $\nu_{0} \in\{-,+\}$. Then $\left(\lambda^{*}, y^{*}\right)$ is a solution of the linear problem (4.19) with $\psi_{n, 0}^{*}(x)$ replaced by $\psi_{0}^{*}(x)$, where $\psi_{0}^{*}(x)$ is defined as the right-hand side of (4.17) with $\left(\lambda_{n, 0}^{*}, y_{n, 0}^{*}\right)$ replaced by $\left(\lambda^{*}, y^{*}\right)$. Since $y^{*} \in S_{k_{0}}^{\sigma_{0}, \nu_{0}}$ it follows that $\lambda^{*}$ is a $k$-th eigenvalue $\lambda_{k}^{*, \sigma_{0}}$ of this problem. Then by (3.1) and Remark 2.3 we get $\lambda^{*} \in J_{k_{0}}^{\sigma_{0}}$. The proof of this lemma is complete.

\section{References}

[1] Z.S. Aliev, On the global bifurcation of solutions of some nonlinear eigenvalue problems for ordinary differential equations of fourth order, Sb. Math. 207 (2016), no. $12,3-29$.

[2] Z.S. Aliyev and Sh.M. Hasanova, Global bifurcation of positive solutions from zero in nonlinearizable elliptic problems with indefinite weight, J. Math. Anal. Appl. 491 (2020), no. 1, 1-14.

[3] Z.S. Aliyev, R.A. Huseynova, Bifurcation in nonlinearizable eigenvalue problems for ordinary differential equations of fourth order with indefinite weight, Electron. $J$. Qual. Theory Differ. Equ. (2017), no. 92, 1-12.

[4] Z.S. Aliyev, L.V. Nasirova (Ashurova), Bifurcation of positive and negative solutions of nonlinearizable Sturm-Liouville problems with indefinite weight, Math. Notes, Miskolc 21 (2020), no. 1, 19-29.

[5] Z.S. Aliyev, H.Sh. Rzayeva, Global bifurcation for nonlinear Dirac problems, Electron. J. Qual. Theory Differ. Equ. (2016), no. 46, 1-14.

[6] L.V. Ashurova (Nasirova), Global bifurcation of solutions for the problem of population modeling, Caspian J. Appl. Math., Ecol. and Econom. 5 (2017), no. 1, 65-71.

[7] H. Berestycki, On some nonlinear Sturm-Liouville problems, J. Diffential Equations 26 (1977), no. 3, 375-390.

[8] S. Cano-Casanova, Compact components of positive solutions for superlinear indefinite elliptic problems of mixed type, Topol. Methods Nonlinear Anal. 23 (2004), no. $1,45-72$.

[9] G. Dai, X. Han, Global bifurcation and nodal solutions for fourth-order problems with sign-changing weight, Appl. Math. Comp. 219 (2013), no. 17, 9399-9407.

[10] G. Dai, R. Ma, Bifurcation from intervals for Sturm-Liouville problems and its applications, Electron. J. Differ. Equ. (2014), no. 3, 1-10. 
[11] M. Delgado, A. Suárez, On the existence and multiplicity of positive solutions for some indefinite nonlinear eigenvalue problem, Proc. Amer. Math. Soc. 132 (2004), no. $6,1721-1728$.

[12] R.S. Cantrell, C. Cosner, Spatial Ecology via Reaction-Diffusion Equations, Ser. Math. Comput. Biol., John Wiley and Sons Ltd., Chichester, UK (2003).

[13] R.A. Fisher, Gene frequencies in a cline determined by selection and diffusion, Biometrics 6 (1950), no. 4, 353-361.

[14] W.H. Fleming, A selection-migration model in population genetics, J. Math. Biology 2 (1975), no. 3, 219-233.

[15] P. Hess, T. Kato, On some linear and nonlinear eigenvalue problems with indefinite weight function, Comm. Partial Differential Equations 5 (1980), no. 10, 999-1030.

[16] E.L. Ince, Ordinary Differential Equations, Dover, New York, 1926.

[17] B. Ko, K. Brown, The existence of positive solutions for a class of indefinite weight semilinear elliptic boundary value problems, Nonlinear Anal. 39 (2000), no. 5, 587597.

[18] L.V. Nasirova, Global bifurcation from intervals of solutions of nonlinear SturmLiouville problems with indefinite weight, Trans. Natl. Acad. Sci. Azerb. Ser. Phys.Tech. Math. Sci. Mathematics 39 (2019), no. 4, 148-154.

[19] P.H. Rabinowitz, Some global results for nonlinear eigenvalue problems, J. Funct. Anal. 7 (1971), no. 3, 487-513.

[20] B.P. Rynne, The structure of Rabinowitz' global bifurcating continua for generic quasilinear elliptic equations, Nonlinear Anal. 32 (1998), no. 2, 167-181.

[21] B.P. Rynne, Bifurcation from zero or infinity in Sturm-Liouville problems which are not linearizable, J. Math. Anal. Appl. 228 (1998), no. 1, 141-156.

[22] K. Schmitt, H. L. Smith, On eigenvalue problems for nondifferentiable mappings, $J$. Differential Equations 33 (1979), no. 3, 294-319.

[23] E. Sovrano, A negative answer to a conjecture arising in the study of selectionmigration models in population genetics, J. Math. Biology 76 (2018), no. 7, 16551672.

[24] K. Umezu, Global bifurcation results for semilinear elliptic boundary value problems with indefinite weights and nonlinear boundary conditions, Nonlinear Differ. Equ. Appl. 17 (2010), no. 3, 323-336.

Leyla V. Nasirova

Sumgait State University, Sumgait AZ5008, Azerbaijan

E-mail address: leyla.ashurova25@gmail.com

Received: August 2, 2021; Revised: November 1, 2021; Accepted: November 5, 2021 